\title{
Spectra of Photobiological Inactivation of SARS-CoV-2 by Solar UVB Radiation (280-320 nm)
}

\author{
V. E. Prokop'eva, $\boldsymbol{b}$, * \\ ${ }^{a}$ Institute of High-Current Electronics, Siberian Branch, Russian Academy of Sciences, Tomsk, 634055 Russia \\ ${ }^{b}$ National Research Tomsk State University, Tomsk, 634050 Russia \\ *e-mail: prokop@ogl.hcei.tsc.ru \\ Received May 11, 2021; revised May 11, 2021; accepted June 7, 2021
}

\begin{abstract}
Primary photoacceptors and spectra of photobiological inactivation of the SARS-CoV-2 molecular structures (genome and S-spike protein) by UVB radiation were determined for the first time based on experimental ground-based observations of the intensity and spectrum of solar UVB radiation in Tomsk $\left(56^{\circ} 29^{\prime} \mathrm{N}, 84^{\circ} 56.89^{\prime} \mathrm{E}\right)$ from March 26, 2020, to March 26, 2021, and the analysis of works on the structure and chemical molecular composition of SARS-CoV-2.
\end{abstract}

Keywords: COVID-19, SARS-CoV-2, spectra of photobiological action, photoacceptor molecules, active centers, RNA, S-protein, guanine, hydroxyl molecule, tryptophan

DOI: $10.1134 / \mathrm{S} 1024856021060191$

\section{INTRODUCTION}

The outbreak of the COVID-19 disease caused by SARS-CoV-2 coronavirus $(\mathrm{CoV})$, which provokes severe acute respiratory syndrome, has put many difficult questions to doctors and the world scientific community about causes and ways and conditions for the spread of the epidemic and has promoted the development and creation of new methods for diagnosis, treatment, and prognosis of the disease.

The full life cycle of viral formations can be divided into two parts: the first includes the penetration, reproduction, and shedding from animal or plant cells (the life cycle of a virus in a cell), the second includes the existence and spread of a virus in an environment. The first part of the life cycle of a virus is studied by virology; therefore, it is not considered in this work. We analyze the second part of the life cycle of viral particles, including routes and conditions for transfer and the ways of survival of viruses in an environment depending on the physical (meteorological) parameters of the latter.

SARS-CoV-2, like many other CoVs, is droplet spread upon contact with patients both with and without symptoms of the disease [1].

An indirect route of transmission is also possible, by contact of a person susceptible to infection with a contaminated object or surface. Disinfecting the environment, including with germicidal ultraviolet (UV) lamps, is of decisive importance for preventing the droplet spread of diseases.
Based on the results of many field and laboratory studies, it has been proved that the bactericidal, cytotoxic, and mutagenic effects of UV radiation are based on the absorption of UV quanta by DNA and RNA molecules of bacteria and viruses [2]. According to the photobiological action, UV radiation is conventionally divided into three wavelength ranges: UVC (100$280 \mathrm{~nm})$, UVB (280-320 nm), and UVA (320$400 \mathrm{~nm}$ ). For disinfection of rooms, UVC mercury lamps with a radiation maximum at a wavelength of $\lambda=253.7 \mathrm{~nm}$ are used. The bactericidal and cytotoxic efficiency of UVC is the highest at $\lambda=250-260 \mathrm{~nm}$ and coincides with the maximal absorption of radiation by nucleic acids.

The results of experiments on exposure of various CoVs to radiation at $\lambda=254 \mathrm{~nm}$ were analyzed in [3] in order to determine the dose of UV radiation required for their inactivation. The coronaviruses had a similar physicochemical structure, the same length of a single RNA strand, and the genome size. (Chinese researchers deciphered and characterized the genome of the new coronavirus and found it to be very similar to a number of already studied CoVs $[4,5]$.) The spectra of photobiological action and the dose of UVC radiation required to reduce the number of viable viruses by $90 \%\left(\sim \mathrm{e}^{-2}\right.$ times $)$ were determined. According to the estimates by the authors, the upper limit of the corresponding dose is $\sim 10.6 \mathrm{~mJ} / \mathrm{cm}^{2}$, while the most likely predicted average value is only $3.7 \mathrm{~mJ} / \mathrm{cm}^{2}$.

Almost simultaneously with [3], a work was published where the possibility of inactivating SARSCoV-2 by UVC radiation was experimentally shown [6]. 
The virus turned out to be very sensitive to UV radiation. In that work, viral material with a high infectious titer was completely inactivated by a few minutes of UV irradiation. The UVC dose required for complete inactivation was $1.048 \mathrm{~mJ} / \mathrm{cm}^{2}$, which was close to the value predicted in [3]. The inactivation effect of UVA radiation $(\lambda=365 \mathrm{~nm})$ was weak.

Thus, the common way of disinfection using UVC radiation [7] ensures inactivation of CoVs, in particular, SARS-CoV-2.

However, under natural conditions, solar UVC radiation is completely absorbed by molecules of ozone, oxygen, and oxides of atmospheric gases already in the upper atmospheric layers. Only solar UVA radiation $(320-400 \mathrm{~nm})$ and attenuated and transformed solar UVB radiation $(280-320 \mathrm{~nm}) \mathrm{com}-$ pletely reach the Earth's surface. Nevertheless, the presence of solar UVB radiation on the Earth's surface and the high probability of its photobiological effect on bacteria and viruses are very interesting.

The principal work in this direction is the message [8], where "lethal" radiation doses are determined, which reduce the viability of viruses by $37 \%$ (e times) depending on the radiation parameters and the size of the virus genome. The results and conclusions of [8] were used as the basis for calculating the lethal doses of solar radiation required for SARS CoV-2 inactivation in different regions of the Earth from January to May 2020 [9].

The survival of SARS-CoV-2 in water-droplet saliva aerosols and on different surfaces under illumination by a source simulating solar UVB radiation (xenon lamp) was shown in a number of laboratory experiments [10-12].

The results of work [8] concerning the spectrum of photobiological action of solar UVB radiation (280$320 \mathrm{~nm}$ ) on viral particles and experimental data on lethal doses of UVC/B radiation [3, 10-12] were used in [13] to calculate the spectra of photobiological action and lethal doses of radiation in different regions of the Earth.

Based on the latest work, simulation models and calculation programs have been created for estimating the decay (inactivation) time of SARS-CoV-2 on surfaces and in aerosols, which mainly depends on the UV index (UVI) and, insignificantly, on the temperature and relative humidity of the environment [14]. The above environmental parameters can be determined for a specific site from general weather resources on the Internet.

In this work, the primary photoacceptors (chromophores) and spectra of photobiological inactivation of molecular structures of SARS-CoV-2 by the UVB radiation present on the Earth's surface are studied based on the results of ground-based observations of the intensity and spectrum of solar UVB radiation in Tomsk ( $56^{\circ} 29^{\prime} \mathrm{N}, 84^{\circ} 56.89^{\prime}$ E) from March 26, 2020, to March 26, 2021, and the analysis of scientific works on the structure and chemical molecular composition of the $\mathrm{CoV}$.

\section{EXPERIMENTAL}

To measure parameters of solar radiation on the Earth, we used two Ocean Optics (OO) HR4000 spectrometers manufactured to our order with a high spectral resolution and a sensitivity ranges $200-300$ and $250-350 \mathrm{~nm}$. A spectral resolution of $\sim 0.1 \pm 0.05 \mathrm{~nm}$ was attained with the use of a Toshiba TCD1304AP linear CCD array consisting of 3648 elements (pixels), a diffraction grating of 2400 lines $/ \mathrm{mm}$, and an entrance slit of $25 \mu \mathrm{m}$. The optical axis of the instrument was manually aligned with the central region of the solar disk using guides. To measure the spectral resolution of the instruments and calibrate by wavelengths, we used the radiation of a DRGS-12 lowpressure mercury lamp. To avoid harmful background illumination of the photosensitive elements of the device, which accompanies the solar radiation range under study, a broadband UFS-1 light filter with a bandwidth of 230-400 $\mathrm{nm}$ from a standard set of light filters was usually mounted at the entrance.

The parameters of the spectrometers used and the SpectraSuite software allow real-time processing of the results and accumulating the signals (exposure) over $10 \mathrm{~ms}$ to $65 \mathrm{~s}$. The initial numerical values of extraterrestrial solar radiation spectra and of absorption spectra of atmospheric gases, liquid water, and biological molecules in aqueous solutions were taken from well-known databases and data banks [15-17]. The geometeorological programs Windy.com and Gismeteo were used for the daily measurements and comparison of meteorological parameters in different regions (temperature, pressure, ozone, humidity, UVI, aerosol, $\mathrm{NO}_{2}$, etc.)

\section{EXPERIMENTAL RESULTS AND DISCUSSION}

\section{Primary Photoacceptors and Molecular Structures of CoV in Saliva Bioaerosols}

The absorption spectra of saliva in health and in pathologies of the oral cavity have been well studied by molecular absorption spectroscopy and fluorescence [18].

It should be said that the chemical composition of water-droplet aerosol particles simulating human saliva containing viral formations is well studied and described in the experimental works [10-12]. Human saliva is $98.5 \%$ water and dissolved inorganic salts and organic molecules (mucin, lysozyme, amylase, maltose enzymes, and more than 100 enzymes of all classes). In the case of COVID-19, SARS-CoV-2 is added to these molecules.

Biological fluids and tissues, including blood, usually weakly absorb in the UVB spectral region, at wavelengths of $310 \pm 10 \mathrm{~nm}$; the absorption is due to 
absorption by protein molecules, nucleic acids and their decay products on the short-wavelength side $(\lambda \leq$ $290 \mathrm{~nm}$ ), and by molecules of porphyrins, carotenes, etc., on the long-wavelength side $(\lambda \geq 320 \mathrm{~nm})$. These organic molecules compete for UVB photons with SARS-CoV-2 molecules, which absorb in the same region; this reduces the efficiency of photobiological inactivation of the virus. The main component of saliva, water, very weakly absorbs UVB radiation; therefore, the spectra of the photobiological action of photoacceptor biological molecules are close to the absorption spectrum of these molecules in aqueous solutions in this region [17]. The conclusion is all the more true for water-droplet bioaerosols of saliva of patients with COVID-19; the average size of these particles does not exceed 100 microns $(0.1 \mathrm{~mm})$ and include $\mathrm{CoVs}$ inactivated by UVB radiation.

Let us consider in detail the molecular composition of SARS-CoV-2. Its genome consists of single-stranded RNA surrounded by a protein-lipid membrane, the most important part of which is the $\mathrm{S}^{\prime}$-protein, responsible for the entry of the virus into a host cell.

In addition to four nitrogenous bases, the RNA chain contains ribose, which has an additional hydroxyl chromophore group $-\mathrm{OH}$ in the 2' carbon position as compared to deoxyribose in the DNA molecule. This group increases the probability of hydrolysis of molecules and determines the physicochemical, including optical and spectroscopic, properties of RNA at wavelengths near the main absorption band $\mathrm{A}^{2} \Sigma^{+}\left(\mathrm{v}^{\prime}=0\right) \leftarrow \mathrm{X}^{2} \Pi\left(\mathrm{v}^{\prime \prime}=0\right) \mathrm{OH}$, which is observed in the form of a relatively narrow band in the region 306.4-315 nm with a maximum at a wavelength near $309 \mathrm{~nm}$ at room temperatures [19]. This leads to a longwavelength shift and an increase in absorption in the UVB region, and, hence, to an increase in the rate of inactivation of the RNA chain as compared to DNA.

In addition to the standard photobiological way of genome inactivation (RNA), SARS-CoV-2 can contain other molecular structures capable of changing their physicochemical properties under the action of solar UVB radiation. Indeed, the physicochemical structure and molecular composition of SARS-CoV-2, as well as its functional properties, are associated, first of all, with protein spikes, the active centers of which are similar to those of SARS-CoV and contain a relatively large amount of aromatic amino acids, such as phenylalanine, tryptophan, and tyrosine [20]. The absorption of photons by these photoacceptors of the active centers of the S-protein can result in rearrangement of the molecular structure of an active center and inactivate $\mathrm{CoV}$. We understand inactivation as violation of the standard ways of binding aromatic amino acid molecules of the active center of the S-protein with the receptor molecules of a host cell.

The last two of the abovementioned aromatic amino acids have a long-wavelength absorption shoulder in the UVB spectral region, due to the absorption of the aromatic (phenolic) ring. Moreover, the tyrosine molecule contains an additional chromophore group, $-\mathrm{OH}$, which shifts the absorption band of the molecule toward long waves in comparison with phenylalanine and tryptophan. Just the presence of tyrosine in a variety of biological tissues causes wellknown photobiological phenomena, including those responsible for the erythema response and skin pigmentation.

The absorption spectra of these photoacceptors, necessary for calculating the spectra of photobiological action in the UVB range, are now well known [1517]. To calculate the spectra of photobiological action, the spectra of solar radiation on the surface of the Earth or habitat should be known in addition to the absorption spectra of photobiological targets. These spectra can be obtained in different ways, for example, from satellite measurements, model calculations, and direct measurements with spectral instruments of a ground-based network. Ground-based measurements are the most accurate, especially for the UVB spectral region [21].

Based on the molecular composition of the genome and membrane of SARS-CoV-2, especially of the S-protein of the membrane, the presence of chromophores in the active center of this protein, including tryptophan and tyrosine, capable of absorbing in the UVB spectral region, shows their sensitivity to solar radiation, which means a possibility of $\mathrm{CoV}$ inactivation.

\section{Seasonal Solar Radiation Spectra on the Earth's Surface (Tomsk)}

The level of UV radiation on the Earth's surface is determined by several well-studied factors: the time of the year and the day, meteorological parameters, and geographical coordinates of an observation site [20, 21].

Figure 1 shows the spectra of midday solar radiation measured in different seasons on characteristic days. These days provide a representative sample of the spectra characterizing different seasons in the region of observation.

The spectral data in Fig. 1 show that the shortwavelength boundary of solar UV radiation measured on the day of the winter solstice of December 21, 2020 (the shortest daylight time and the minimal solar angle), falls on $\sim 310 \mathrm{~nm}$. Under moderate transparency and average ozone content, this boundary coincides with the short-wavelength boundary of direct solar radiation on the Earth measured in $[20,21]$ at a solar angle of $15^{\circ}$. The radiation intensity at the longwavelength boundary of the UVB range $(\lambda=320 \mathrm{~nm})$ decreases by two orders of magnitude in comparison with the solar radiation vertically incident on the Earth's surface [20, 21]. This boundary shifts to $295 \mathrm{~nm}$ on the day of the summer solstice of June 21, 2020 (a clear day), and the radiation intensity near the 


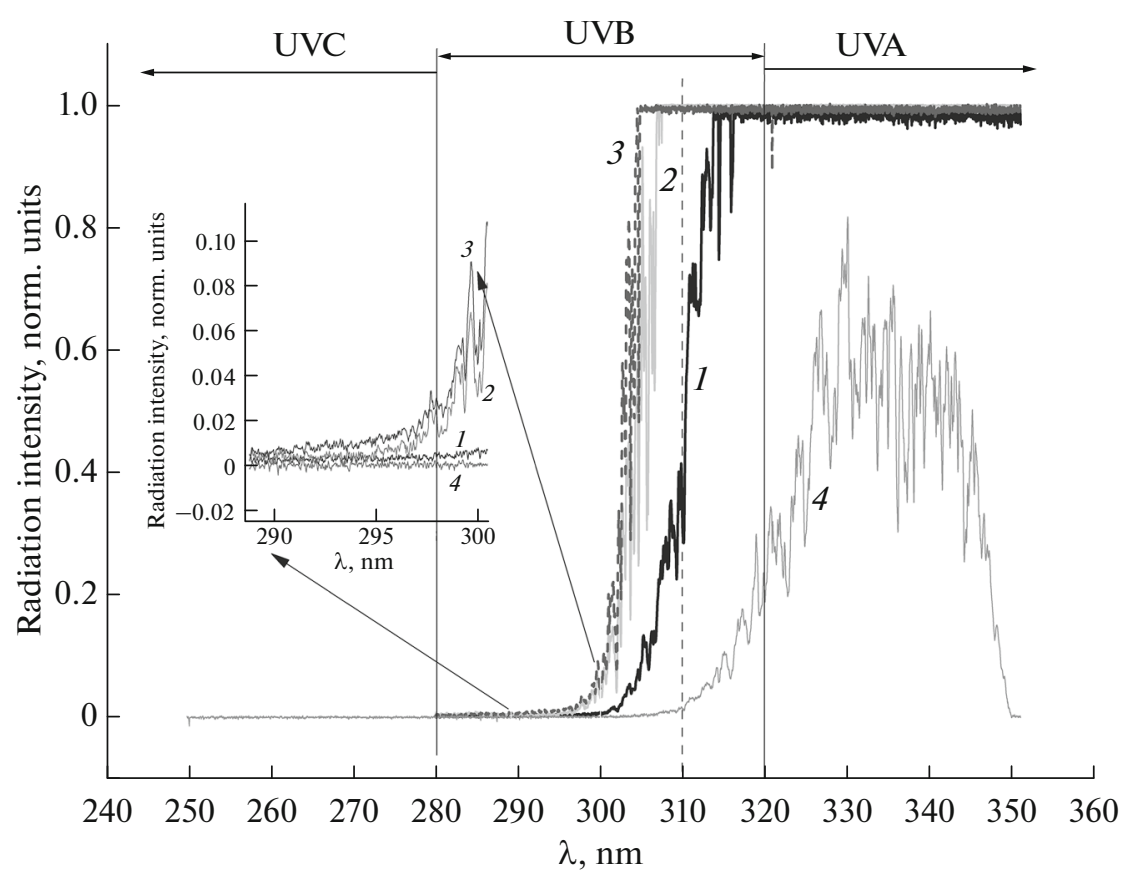

Fig. 1. Solar radiation spectra: in spring (March 20, 2021, spring equinox) (curve 1); in summer (June 21, 2020, summer solstice) (curve 2); in autumn (September 22, 2020, autumnal equinox (curve 3); in winter (December 21, 2020, winter solstice) (curve 4); the fragment shows the short-wavelength part of the spectrum in the region $290-300 \mathrm{~nm}$.

long-wavelength boundary $(\lambda=320 \mathrm{~nm})$ increases by $1-2$ orders of magnitude as compared to the day of the winter solstice.

As can be seen from Fig. 1, the differences in the intensity of direct solar UVB radiation and in the short-wavelength boundaries (310 and $295 \mathrm{~nm}$ ) are maximal on the days of the summer and winter solstices. Another well-known feature that we have ascertained is that scattered solar radiation at moderate and small solar angles makes a much larger contribution to the total (global) radiation than the direct radiation, especially solar UVB radiation.

One can see in Fig. 1 that the short-wavelength boundaries of the midday solar radiation spectra on the days of spring and autumn equinox significantly differ and equal 300 and $\sim 292 \mathrm{~nm}$, respectively. The latter value turned out to be even lower than on the day of the summer solstice. Since the solar angle is the same on these days, the significant differences in the short-wavelength boundaries and in the UV radiation intensity in these seasons in Tomsk can be explained by the difference in the total ozone (TO) $[\mathrm{X}]$ in summer-autumn ([X] minimum) and winter-spring ([X] maximum) periods [21, 22].

Based on the data obtained, it is possible to qualitatively estimate the ultraviolet index (UVI) for different seasons of 2020-2021. UVI reflects the erythema response of human skin (redness, burns, and pigmentation) and is reported in the weather forecast for western Siberia, including Tomsk. UVI is minimal in the cold season and is usually zero in late autumn
(November) and in winter (December, January, and February). At this time of the year, epidemiological diseases tend to emerge and spread in the Northern Hemisphere. For example, the first case of COVID-19 was officially recorded in China, Wuhan $\left(30^{\circ} 35^{\prime} \mathrm{N}\right)$, in December 2019; the COVID-19 morbidity rate peak fell on December 21-25, 2020, in Russia and Tomsk.

As follows from Gismeteo data, UVI was maximal $(\sim 4-8)$ in summer and early autumn (September) in 2020. Therefore, the rate of droplet spread of the virus was limited due to the high degree of disinfection of the environment by solar UVB radiation.

\section{Identification of Solar Radiation Spectrum on the Earth's Surface (Tomsk)}

In contrast to the near-constant spectrum and intensity of extraterrestrial solar radiation, which has been studied with a high spectral resolution and in a wide spectral range, the terrestrial spectra of solar UVB radiation are little studied, especially in high latitudes, due to low intensities, variability, and dependence on meteorological parameters, which complicates the interpretation and identification of the UVB spectrum on the Earth's surface (Fig. 2).

To identify the spectra of solar UVB radiation on the Earth's surface, we measured the solar radiation spectra in Tomsk (curve 2 in Fig. 2) and matched them with the standard spectra of extraterrestrial solar radiation (curve 1 in Fig. 2, MoldRur data for 2010) recorded in [23] with a high spectral resolution of 


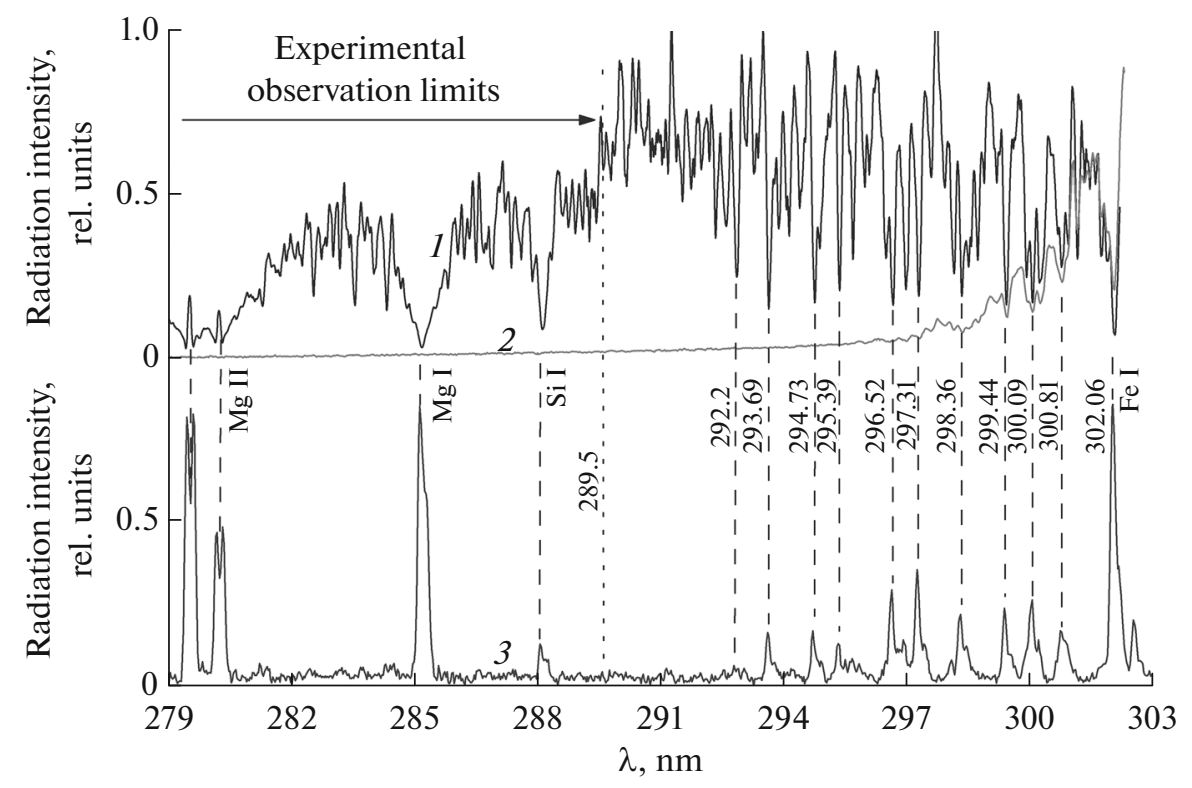

Fig. 2. High-resolution spectra of extraterrestrial solar radiation according to data [23] (curve 1) and of solar radiation on the Earth's surface (Tomsk) on August 30, 2020 (curve 2); spectrum of emission of a sample of whole blood in the gas-discharge plasma of a carbon arc (curve 3).

$0.0028 \mathrm{~nm}$, as well as with the arc plasma emission spectra of dry samples of whole blood (curve 3 in Fig. 2). Spectra 2 and 3 were recorded in the present work on a HR4000 spectrometer with a spectral resolution of $0.1 \mathrm{~nm}$ in the range $250-350 \mathrm{~nm}$. The high spectral resolution of the HR4000 spectrometer made it possible to identify the solar spectra on the Earth's surface in the spectral region $302.06 \pm 290 \mathrm{~nm}$ with an accuracy matching the spectral maxima and minima of no worse than $\pm 0.03 \mathrm{~nm}$. The analysis of the emission spectrum recorded in this range has shown it to be completely identical to the spectrum of extraterrestrial solar radiation at $\lambda=294.8 \mathrm{~nm}$ and to be transformed as a result of absorption by ozone and oxygen molecules, impurity molecules, and aerosol particles, including those of biological origin, at $\lambda=294.8-$ $289.5 \mathrm{~nm}$.

We also measured the spectra of direct and scattered solar radiation in the fall season, up to December 29,2020 . The general result of those measurements on cloudy days, the total number of which exceeds $90 \%$ in that time, was the shift of the short-wave solar radiation boundary to $310-320 \mathrm{~nm}$ in the end of December. That was caused by small sun angles, strong backscattering, and UVB absorption by clouds. However, when measuring on clear days, which occurred in the end of November (November 26-29, 2020), the situation strongly changed: the short-wavelength boundary of direct solar radiation was equal to $310 \mathrm{~nm}$ at noon and reached $296 \mathrm{~nm}$ when the sun was at the zenith and at zenith angles $<45^{\circ}$ due to the Rayleigh effect. This value is close to the limiting summer values of $\sim 292 \mathrm{~nm}$. The boundary increased with the zenith angle. Although the intensities of the scattered midday solar radiation were orders of magnitude lower than the summer values, the sky- and time-integral short-wave solar radiation corresponded to UVI $\approx 3-4$ on clear days in autumn-spring even at the latitude of Tomsk $\left(56^{\circ} \mathrm{N}\right)$, which ensured inactivation of $\mathrm{CoV}$ even in winter. It is clear that, along with strong solar UVB radiation, the inactivation of $\mathrm{CoV}$ could also be due to a critical decrease in the ambient temperature by $25-30^{\circ} \mathrm{C}[2,3]$.

\section{Spectra of Photobiological Action}

Thus, based on the spectra of solar radiation on the Earth's surface measured during a year (March 3, 2020-March 26, 2021) in Tomsk and determination of the primary photoacceptor molecules of SARSCoV-2 $[4,5,20]$ and their absorption spectra, it is possible to calculate the spectra of photobiological inactivation of this coronavirus.

Figure 3 shows the spectra of the photobiological inactivation of SARS-CoV-2 by solar UVB radiation calculated with and without accounting for the contribution of the hydroxyl chromophore OH to the RNA absorption spectrum, as well as the spectrum of photobiological inactivation of the S-protein due to absorption by tryptophan molecules.

The spectra of photobiological action were calculated by the equation $A(\lambda)=I(\lambda) K(\lambda)$, where $I(\lambda)$ is the solar radiation intensity at a certain time point (in our case, 12:58 LT of December 17, 2020) normalized to the wavelength $320 \mathrm{~nm} ; K(\lambda)$ is the absorption coefficient of a certain photoacceptor (an active center which induces the corresponding photobiological 


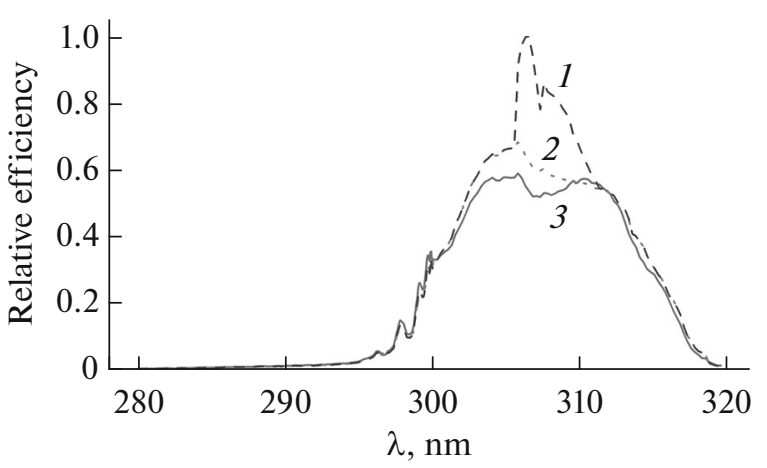

Fig. 3. Spectra of photobiological inactivation of SARSCoV-2 taking account of the glucose $\mathrm{OH}$ molecule in the 2' carbon position (RNA) (1); guanine nitrogenous base (RNA) (2); tryptophan (spike S'-protein) (3); December 17, 2020, 12:58 LT.

effect) normalized to the absorption coefficient at a wavelength of $300 \mathrm{~nm}$.

We assumed independent absorption of photons by individual photoacceptors in molecular nanostructures with dimensions equal to or less than the wavelength of the incident light (active center of S-protein, RNA). When calculating the spectrum of photobiological inactivation of RNA considering the $\mathrm{OH}$ molecule, we assumed the absorption cross section of the hydroxyl molecule at the band maximum $(\sim 306.4 \mathrm{~nm})$ to be equal to the absorption cross section of guanine in the same wavelength range. However, for correct assessment of the spectra of photobiological inactivation of RNA, the high-resolution $(\leq 1 \mathrm{~nm})$ measurements of the absorption cross section of the $\mathrm{OH}$ functional group in different complex biological molecules and structures are required.

Normalization of $K(\lambda)$ (for wavelengths shorter than $300 \mathrm{~nm}$ ) assumes that each absorbed photon at wavelengths $<300 \mathrm{~nm}$ causes this photobiological effect with $100 \%$ quantum efficiency. From the point of view of estimation of the significance of solar UVI for photobiological phenomena, the lethal dose and time of destructive processes, for example, inactivation (bactericidal effect) of viruses or microbes, or of constructive processes or a positive effect, for example, the synthesis of vitamin $\mathrm{D}$, are important. The daily dose, which is calculated by integrating over the entire daylight period, as in the case of photosynthesis, is often the most important for estimating the significance of solar radiation.

As follows from the numerical estimates of the spectra of photobiological action, the inactivation efficiency is maximal at wavelengths of $300-315 \mathrm{~nm}$, which coincides with the maximal intensity of solar UVB radiation on clear days. Hence, gas-discharge quartz lamps with water vapor, with high-intensity emission at wavelengths of 306.4-320 nm, and semiconductor LEDs emitting in the same range, can be effective and safe for the prevention and disinfection of indoor air and various surfaces.

\section{CONCLUSIONS}

The ground-based experimental measurements of the spectrum of direct solar UVB $(280-320 \mathrm{~nm})$ radiation were carried out with a high spectral resolution $(\sim 0.1 \pm 0.05 \mathrm{~nm})$ in Tomsk $\left(56^{\circ} 29^{\prime} \mathrm{N}, 84^{\circ} 56^{\prime} \mathrm{E}\right)$ from March 26, 2020, to March 26, 2021. It was found that the short-wavelength boundary of direct solar radiation shifts to $310 \mathrm{~nm}$, on both cloudy and clear days in late September and winter, while this boundary of the scattered and global radiation of the sky can reach 296 $\mathrm{nm}$ on clear days due to Rayleigh scattering of direct solar radiation in the upper atmosphere.

Based on experimental studies of the spectral parameters of biological tissues (saliva and human whole blood) and the analysis of the literature devoted to the chemical molecular composition of the coronavirus, we determined the primary photoacceptors:

- Guanine-the nitrogenous base of the RNA chain of the SARS-CoV-2.

- Hydroxyl molecules-OH glucose in the carbon 2 ' position in the RNA molecule.

- Tryptophan-in the active center of S-protein (spike glycoprotein $\mathrm{S}$ ) of the coronavirus.

Experimental data on the spectrum of solar UVB radiation during the spring-autumn in Tomsk and the absorption spectra of primary photoacceptors of $\mathrm{CoV}$ made it possible to calculate the spectrum of photobiological inactivation of SARS-CoV-2 in saliva aerosols.

Based on the analysis of recent scientific publications and the results of this study, a conclusion is drawn that the rate of increase in the number of cases of COVID-19 largely depends on the disinfection of the $\mathrm{CoV}$ transfer medium by ultraviolet solar radiation (UVB and UVA) in a region. The intensity and spectrum of UV radiation is largely determined by the geophysical and meteorological parameters of the region, which, in turn, depend on the season and latitude.

\section{ACKNOWLEDGMENTS}

The author is grateful to V.V. Udut (National Research Medical Center, Russian Academy of Sciences, Tomsk), N.E. Zueva and V.V. Zuev (Institute of Monitoring of Climatic and Ecological Systems, Siberian Branch, Russian Academy of Sciences, Tomsk) for well-meaning criticism and discussion of the results of the work.

\section{CONFLICT OF INTEREST}

The author declares that he has no conflicts of interest. 


\section{REFERENCES}

1. R. Xu, B. Cui, X. Duan, P. Zhang, X. Zhou, and Q. Yuan, "Saliva: potential diagnostic value and transmission of 2019-nCoV,” Int. J. Oral. Sci. 12 (11), 1-6 (2020).

2. B. L. Diffey, "Solar ultraviolet radiation effects on biological systems,” Phys. Med. Biol. 36, 299 (1991).

3. M. Hessling, K. Hones, P. Vatter, R. Haag, and N. Sieber, "Ultraviolet irradiation doses for coronavirus inactivation-review and analysis of coronavirus photoinactivation studies," GMS Hyg. Infect. Control 15, 1-8 (2020).

4. R. Lu, X. Zhao, Ju. Li, P. Niu, B. Yang, H. Wu, W. Wang, H. Song, B. Huang, N. Zhu, Yu. Bi, X. Ma, F. Zhan, L. Wang, T. Hu, H. Zhou, Z. Hu, W. Zhou, L. Zhao, Ji. Chen, Ya. Meng, Ji. Wang, Ya. Lin, Ji. Yuan, Z. Xie, Ji. Ma, W. J. Liu, D. Wang, Xu W. Wenbo, E. C. Holmes, G. F. Gao, G. Wu, W. Chen, W. Shi, and W. Tan, "Genomic characterisation and epidemiology of 2019 novel coronavirus: implications for virus origins and receptor binding," Lancet 395 (10224), 565-574 (2020).

5. F. Wu, S. Zhao, B. Yu, Yan-Mei Chen, W. Wang, Yi. Hu, Zhi-Gang Song, Zhao-Wu Tao, Jun-Hua Tian, Yuan-Yuan Pei, Ming-Li Yuan, Yu-Ling Zhang, FaHui Dai, Yi Liu, Qi-Min Wang, Jiao-Jiao Zheng, L. Xu, Ed. C. Holmes, and Yong-Zhen Zhang, "Complete genome characterisation of a novel coronavirus associated with severe human respiratory disease in Wuhan, China," BioRxiv (2020). https://doi.org/10.1101/2020.01.24.919183

6. C. S. Heilingloh, U. W. Aufderhorst, L. S. Schipper, U. Dittmer, O. Witzke, D. Yang, X. Zheng, K. Sutter, M. Trilling, M. Alt, E. Steinmann, and A. Krawczyk, "Susceptibility of SARS-CoV-2 to UV irradiation," Am. J. Infect. Control 48, 1273 (2020).

7. E. A. Sosnin, M. V. Erofeev, S. M. Avdeev, A. N. Panchenko, V. A. Panarin, V. S. Skakun, V. F. Tarasenko, and D. V. Shitts, "An ultraviolet barrier-discharge $\mathrm{OH}$ molecular lamp, Quantum Electron. 36 (10), 981-983 (2006).

8. C. D. Lytle and J.-L. Sagripanti, "Predicted inactivation of viruses of relevance to biodefense by solar radiation," J. Virol. 79 (22), 14244-14252 (2005).

9. F. Nicastro, G. Siron, E. Antonello, A. Bianco, M. Biasin, J. R. Brucato, I. Ermolli, G. Pareschi, M. Salvati, P. Tozzi, D. Trabattoni, and M. Clerici, "Modulation of COVID-19 epidemiology by UV-B and -A photons from the Sun," MedRxiv (2020).

doi 10. 1101/2020.06.03.20121392

10. M. Schuit, S. Gardner, S. Wood, K. Bower, G. Williams, D. Freeburger, and P. Dabisch, "The influence of simulated sunlight on the inactivation of influenza virus in aerosols," J. Infect. Dis. 221, 372-378 (2019).

11. M. Schuit, S. Ratnesar-Shumate, YolitzJ. Jason, G. Williams, W. Weaver, B. Green, D. Miller, M. Krause, K. Beck, S. Wood, B. Holland, Jo. Bohannon, D. Free- burger, I. Hooper, J. Biryukov, L. A. Altamura, V. Wahl, M. Hevey, and P. Dabisch, "Airborne SARS-CoV-2 is rapidly inactivated by simulated sunlight," J. Infect. Dis. 222, 564-571 (2020).

12. S. Ratnesar-Shumate, G. Williams, GreenB. Brian, M. Krause, B. Holland, St. Wood, Jo. Bohannon, Je. Boydston, D. Freeburger, I. Hooper, K. Beck, Jo. Yeager, LouisA. Altamura, Je. Biryukov, Ja. Yolitz, M. Schuit, V. Wahl, M. Hevey, and P. Dabisch, "Simulated sunlight rapidly inactivates SARS-CoV-2 on surfaces," J. Infect. Dis. 222, 214-222 (2020).

13. J. Herman, B. Biegel, and L. Huang, "Inactivation times from 290 to $315 \mathrm{~nm}$ UVB in sunlight for SARS coronaviruses $\mathrm{CoV}$ and $\mathrm{CoV}-2$ using OMI satellite data for the sunlit Earth," Air Qual., Atmos. Health (2020). https://doi.org/10.1007/s11869-020-00927-2

14. Estimated Decay of SARS-CoV-2 (virus that causes COVID-19). www.dhs.gov/science-and-technology/sarscalculator. Cited April 11, 2021.

15. C. A. Gueymard, "Reference solar spectra: Their evolution, standardization issues, and comparison to recent measurements," Adv. Space Res. 37, 323-340 (2006).

16. H. Keller-Rudek, G. K. Moortgat, and R. Sander, "The MPI-Mainz UV/VIS spectral atlas of gaseous molecules of atmospheric interest," Earth Syst. Sci. Data 5 (2), 365-373 (2013).

17. S. Jacques and S. Prahl, Optical absorption of water compendium. https://omlc.org/spectra/water/abs/index. html. Cited September 9, 2020.

18. F. Yazid, M. N. Zain, Z. M. Yusof, F. S. Ghazali, S. A. Zulkifli, N. M. Nadri, S. H. Z. Ariffin, and R. M. A. Wahab, "Caries detection analysis in human saliva alpha amylase," AIP Conf. Proc. 2203, 1-7 (2020).

19. K. P. Huber and G. Herzberg, Molecular Spectra and Molecular Structure. IV. Constants of Diatomic Molecules (Springer, 1979).

20. M. A. Tortorici, A. C. Walls, Y. Lang, Chu. Wang, Z. Li, D. Koerhuis, G.-J. Boons, B.-J. Bosch, F. A. Rey, R. J. Groot, and D. Veesler, "Structural basis for human coronavirus attachment to sialic acid receptors,” Nat. Struct. Mol. Biol. 26, 481-489 (2019).

21. Bioindication of Stratospheric Ozone, Ed. by V.V. Zuev (Publishing House of SB RAS, Novosibirsk, 2006) [in Russian].

22. B. D. Belan, Tropospheric Ozone (Publishing House of SB RAS, Tomsk, 2010) [in Russian].

23. J. L. Kohl, W. H. Parkinson, and R. L. Kurucs, Center and Limb Solar Spectrum in High Spectral Resolution: $225.2 \mathrm{~nm}$ to $319.6 \mathrm{~nm}$ (Harvard-Smithsonian Centre for Astrophysics, Cambridge, 1978).

Translated by $O$. Ponomareva 